\title{
Photonic analogies of gravitational attractors
}

\author{
Damian P. San-Roman-Alerigi, Tien K. Ng, \\ Ahmed B. Slimane, Yaping Zhang, \\ and Boon S. Ooi. \\ Photonics Laboratory, \\ Division of Compuert, Electrical and Mathematical Sciences, \\ King Abdullah University of Science and Technology (KAUST) \\ Saudi Arabia. \\ Email: boon.ooi@kaust.edu.sa
}

\author{
Mohammad Alsunaidi \\ Department of Electrical Engineering \\ King Fahd University of Petroleum and Minerals \\ Saudi Arabia \\ Email: msunaidi@kfupm.edu.sa
}

\begin{abstract}
In our work we demonstrate a Gaussian-like refractive index mapping to realize light trapping. Our study shows that this centro-symmetrical photonic structure is able to mime the light geodesics described by celestial mechanics. Possible applications are discussed.
\end{abstract}

\section{INTRODUCTION}

Einstein's general relativity theory allows us to understand the dynamics of mass and massless particles as they travel through a space-time that is fill with matter. From it we learn that matter and the geometry of space-time are intimately related; matter changes the curvature the metric, of spacetime. This alteration in the very fabric of space-time in turn curves the geodesics, the space-time paths of the particles; and gives birth to what we perceive as gravity. For example in the gravitational lensing effect, depicted in figure 1a, photons travelling near a massive star are deflected from their otherwise straight path due to the curvature of space-time by the massive body in their vicinity. Another illustration of this effect comes from spinning black holes, which have been shown recently to trap photons in a manifold of orbits around them as a function of the initial conditions and the shape of the spinning black hole [1], see figures $1 \mathrm{~b}$ and $1 \mathrm{c}$.

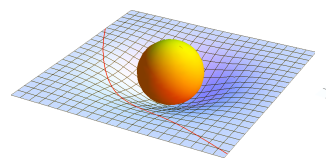

(a)

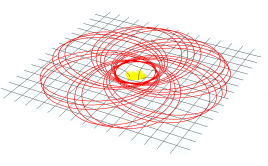

(b)

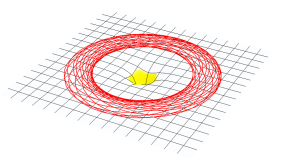

(c)
Fig. 1. Photon's path in the vicinity of a star (a), and (b)-(c) around a spinning black hole at different initial conditions.

When comparing the latter examples to the refraction of light by a heterogeneous medium, one may wonder if it would be possible to fabricate an optical analogue to the celestial geodesics by controlling the refractive index stepwise. Specific to this letter are those which describe an orbital movement. If viable, they could enable a new variety of photonic devices ranging from light traps to concentrators, and optical delays; to name a few.

\section{DERIVING THE MODEL}

In celestial mechanics the geodesic of a photon travelling in a space with coordinates $\left(x^{0}, x^{1}, x^{2}, x^{3}\right)$, where $x^{0}$ corre- sponds to time, and $\vec{r}=\left(x^{1}, x^{2}, x^{3}\right)$ to space; metric tensor $g_{i j}$, and line element $d s^{2}=g_{i j} d x^{i} d x^{j}$; is the solutions to the Lagrange-Euler equation, with Lagrangian:

$$
L=\frac{1}{2}\left[g_{00} \frac{\partial x^{0}}{\partial \lambda}-g_{i j} \frac{\partial x^{i}}{\partial \lambda} \frac{\partial x^{j}}{\partial \lambda}\right],
$$

where the derivatives are taken with respect to the affine parameter $\lambda$. The physical description behind equation 1 is of particular interest to optics, if the metric was related to the electromagnetic tensors, then it could be possible to write the lightpath in the paraxial approximation as a function of either the refractive index $n=f\left(x^{0}, \vec{r}\right)$ or the metric, hence it could be possible to use Fermat's principle, where equation 1 would take the role of an optical Lagrangian. Such relation is given by transformation optics [2], [3], [4], [5], by virtue of conformal mappings it makes possible to build a link between the curvilinear relativistic space-time and the optical electromagnetic properties of a [meta]material.

Recently Genov et al., and us, independently showed that if the line element of space-time takes the form $d s^{2}=$ $g_{00} d x^{0} d x^{0}-g_{i i} d x^{i} d x^{i}$, it is possible to map the curved spacetime to an isotropic, non-dispersive and non-absorbing medium [6], [7]:

$$
n=\varepsilon=\mu=\frac{\sqrt{-g} \cdot g^{i j}}{g_{00}},
$$

where $g=\operatorname{det}\left(g_{i j}\right)$. Furthermore if we consider a static medium we can make use the Minkowski spacetime of special relativity, where the tensor $g$ is diagonal, with entries $g=\operatorname{diag}(1,-1,-1,-1)$ and line element: $d s^{2}=c\left(d x^{0}\right)^{2}-$ $d x^{i} d x^{i}, \quad i=1,2,3$, then by means of equation 2 we can derive the refractive index $n=n(\vec{r})$, and setting the affine parameter $\lambda$ as $x^{0}$, i.e. $\lambda=x^{0}=t$ obtain the Lagrange-Euler's equations for the massless particle from equation 1 :

$$
n \frac{d^{2} x^{i}}{d t^{2}}=\frac{\partial n}{\partial x^{i}}\left(\frac{d x^{j}}{d t} \frac{d x^{j}}{d t}-\frac{d x^{i}}{d t} \frac{d x^{i}}{d t}\right)-2 \frac{\partial n}{\partial x^{j}} \frac{d x^{j}}{d t} \frac{d x^{i}}{d t} .
$$

Equation 3 describes the parametrized path of the photon subject the aforementioned conditions. The problem then reduces to find a non-singular $n(\vec{r})$ such that the geodesics described by equation 3 are analogous to that of a gravitational attractors, i.e. describe an orbital dynamic. Given certain energy-momentum conditions at the insertion point we found 


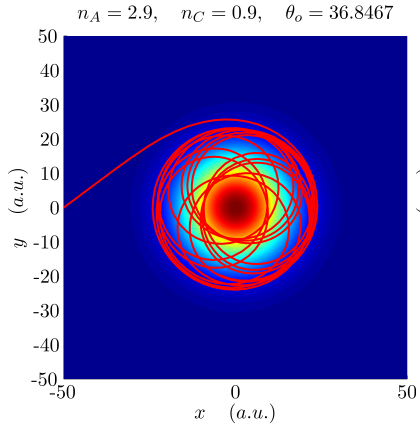

(a)

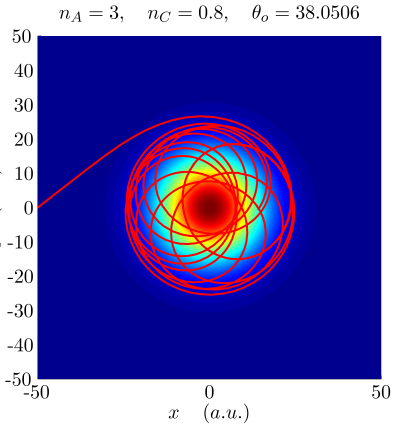

(b)

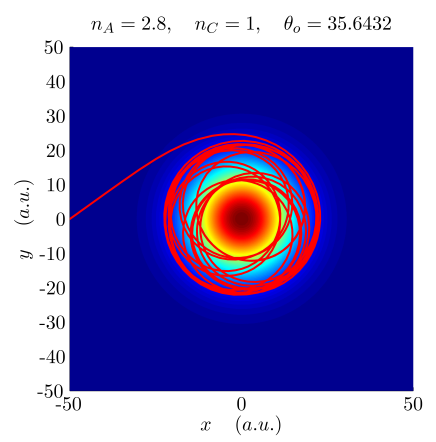

(c)

Fig. 2. Light path (red) in a $2 \mathrm{D}$ Gaussian refractive index profile at different insertion angles.

that a Gaussian-like refractive index $n(\vec{r})=n_{A} e^{-r^{2} / \sigma^{2}}+n_{C}$ yields trapping geodesics for a photon.

\section{Results AND Discussion}

The aforementioned refractive index can be constrain to fabrication limitation[?], [?], [?]. In our case we consider a maximum $n=3.8$ and minimum $n=1$. The results, depicted in figure 2, show that given an initial launch angle between 29.1 and 29.2 degrees for the photon it is possible to trap it around the point of maximum refractive index. In this case the refractive index plays the role of a gravitational attractor; like in the spinning black hole, the motion of the photon is an open orbit and is highly sensitive to modifications in the refractive index.

As it is apparent from figure 2, we found that trapping is highly sensitive to the refractive index distribution perturbations $\Delta n>0.05$, and to the insertion angle, where variations in the order of $\sim 10^{-3}$ modify the trapping orbit. Yet small perturbations, $\Delta n<0.01$, do not change the overall behaviour of the trap. This sensitivity is an interesting opportunity and a key feature to explore dynamic trapping, both important to optical memory and time delays.

Moreover, centro-symmetrical distributions for light can be produce an orbit manifold that not only concerns trapping but deflection of light. Analogous to what light experiences when travelling in the vicinity of a massive object, refractive index distribution such as the one described earlier can used to steer the light path achieving different coupling scenarios as shown in figure ??.

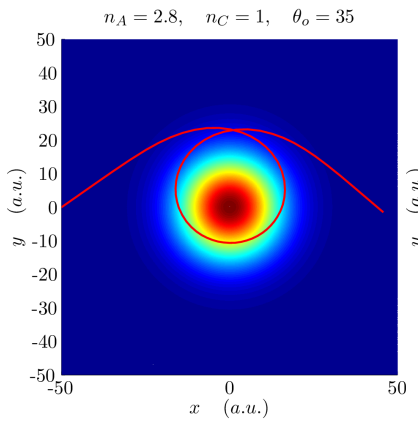

(a)

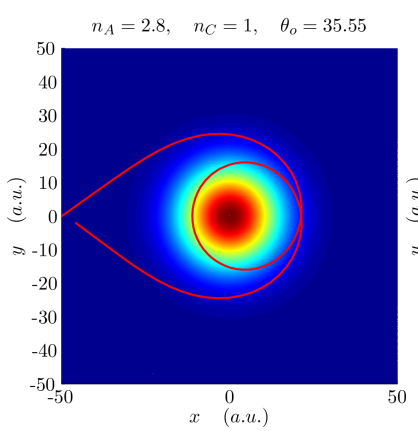

(c)

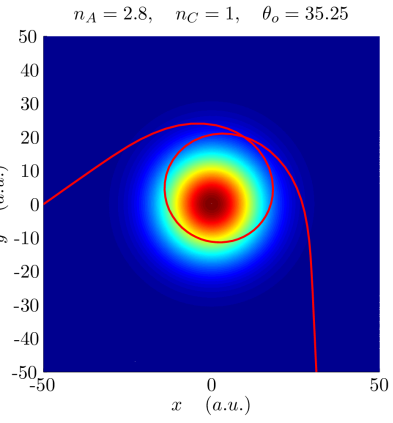

(b)

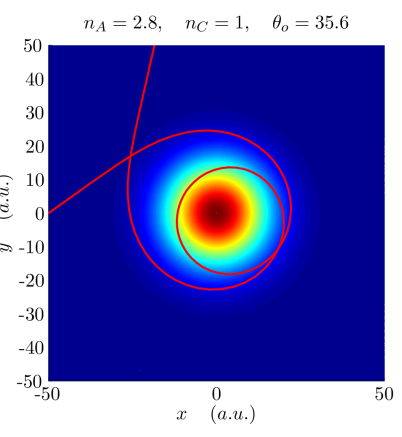

(d)
Fig. 3. Gaussian refractive index distribution used as a beam steerer, analogous to light travelling in the vicinity of a massive object.

Centro-symmetrical refractive index like the one just described brings an interesting opportunity since they can be fabricated for example by stacking different photo-refractive [meta]materials in multiple layers. Recall that in photorefraction the optical properties of the material, permeability and permittivity, change as a function of the light intensity, allowing the fabrication of a continuously varying refractive index as the one required by a Gaussian-like attractor [8], [?]. Moreover, since photo-refractivity is elastic, it allows to modify the refractive index of the layers combined in time, thence enabling or disabling the trap at will.

\section{REFERENCES}

[1] J. Levin and G. Perez-Giz, "A periodic table for black hole orbits," Physical Review D, vol. 77, no. 10, p. 103005, 2008. [Online]. Available: http://prd.aps.org/abstract/PRD/v77/i10/e103005

[2] U. Leonhardt and T. G. Philbin, "General relativity in electrical engineering," New Journal of Physics, vol. 8, no. 10, pp. 247-247, Oct. 2006. [Online]. Available: http://stacks.iop.org/13672630/8/i=10/a=247? key=crossref.4e433b9506a5936770423817211714f3

[3] J. B. Pendry, D. Schurig, and D. R. Smith, "Controlling electromagnetic fields." Science (New York, N.Y.), vol. 312, no. 5781, pp. 1780-2, Jun. 2006. [Online]. Available: http://www.ncbi.nlm.nih.gov/pubmed/16728597

[4] U. Leonhardt and T. T. G. Philbin, "Transformation optics and the geometry of light," Progress in Optics, vol. 53, pp. 69-152, 2009. [Online]. Available: http://linkinghub.elsevier.com/retrieve/pii/S0079663808002023 http://www.sciencedirect.com/science/article/pii/S0079663808002023

[5] H. Chen, C. T. Chan, and P. Sheng, "Transformation optics and metamaterials." Nature materials, vol. 9, no. 5, pp. 387-96, May 2010. [Online]. Available: http://www.ncbi.nlm.nih.gov/pubmed/20414221 
[6] D. A. Genov, S. Zhang, and X. Zhang, "Mimicking celestial mechanics in metamaterials," Nature Physics, vol. 5, no. 9, pp. 687-692, Jul. 2009. [Online]. Available: http://www.nature.com/doifinder/10.1038/nphys1338

[7] D. P. San-Román-Alerigi, A. B. Slimane, T. K. Ng, M. Alsunaidi, and B. S. Ooi, "A possible approach on optical analogues of gravitational attractors," Optics Express, vol. 21, no. 7, p. 8298, Mar. 2013. [Online]. Available: http://www.opticsexpress.org/abstract.cfm?URI=oe-21-7-8298

[8] D. P. San-Roman-Alerigi, D. H. Anjum, Y. Zhang, X. Yang, A. Benslimane, T. K. Ng, M. N. Hedhili, M. Alsunaidi, and B. S. Ooi, "Electron irradiation induced reduction of the permittivity in chalcogenide glass (As2S3) thin film," Journal of Applied Physics, vol. 113, no. 4, p. 044116, 2013. [Online]. Available: http://link.aip.org/link/JAPIAU/v113/i4/p044116/s1\&Agg=doi 